Verbindung, wie schon oben angegeben worden, $\mathrm{C}_{4} \mathrm{H}_{3} \ddot{\epsilon}$ +\#̈̈y; kann also gleich der holländischen Flüssigkeit betrachtet werden, in welcher das Chlor durch das zusammengesetzte Radikal Rhodan ersetzt ist. Es müsste demnach der Körper $\mathrm{C}_{4} \mathrm{H}_{3} \ddot{\mathrm{E}} \mathbf{y}$ isolirt werden können, was mit Kali nicht leicht $\mathrm{zu}$ erreichen ist, weil ein Ueberschuss desselben zersetzend einzuwirken scheint. Vorläufig wurde eine alkoholische Lösung von $\mathrm{C}_{4} \mathrm{H}_{3} \in l$ mit Rhodankalium behandelt und eine senfartig riechende Flüssigkeit erhalten, aus welcher jedoch bis jetzt nichts isolirt werden konnte. Ferner ist es von Interesse, die dem Senföl entsprechenden Verbindungen mit Schwefelquecksilberchlorid und Platinchlorid, ausserdem die durch Ammoniak damit hervorgebrachten Produkte darzustellen, ebenfalls die Existenz einer entsprechenden Verbindung des Senföls mit Rhodanwasserstoffsäure nachzuweisen, worüber die Arbeiten fortgesetzt werden.

\title{
XXIV.
}

\section{Ueber die Einwirkung des Anilins auf Isa-} tin, Bromisatin und Chlorisatin.

\section{Von}

\section{A. Engolhardt. \\ (Bullet. de St. Petersbourg.)}

Bei der Einwirkung von trocknem Ammoniak auf eine Lösung ron Isatin in wasserfreiem Weingeist erhielt Laurent, wie bekannt, Imesatin, dessen Bildung folgendermassen ausgedrưkckt wird:

$$
\underbrace{\mathrm{C}_{16} \mathrm{H}_{3} \mathrm{NO}_{4}}_{\text {Isatin. }}+\mathrm{NH}_{2}-\mathrm{H}_{2} \mathrm{O}_{2}=\underbrace{\mathrm{C}_{16} \mathrm{H}_{6} \mathrm{~N}_{2} \mathrm{O}_{2}}_{\text {Imesatin. }} \text {. }
$$

Ich machte dieselbe Reaction mit Anilin und erhielt bei der Einwirkung von Anilin auf Isatin, Bromisatin und 
Chlorisatin, dem Imesatin ähnliche Verbindungen. Diese Verbindungen, welche ich Phenyl-Imesatin, Phenyl-Bromimesatin und Phenyl-Chlorimesatim nenne, sind Copulationen des Isatins, Bromisatins und Chlorisatins mit Anilin, unter $\mathbf{A b}$ scheidung eines Atoms Wasser $\left(\mathrm{H}_{2} \mathrm{O}_{2}\right)$ und $\mathrm{zwar}$ :

1) Phenyl-Imesatio

$$
=\mathrm{C}_{28} \mathrm{H}_{10} \mathrm{~N}_{2} \mathrm{O}_{2}=\mathrm{C}_{16} \mathrm{H}_{5} \mathrm{NO}_{5}+\mathrm{C}_{12} \mathrm{H}_{7} \mathrm{~N}-\mathrm{H}_{2} \mathrm{O}_{2} \text {. }
$$

2) Phenyl-Bromimesatin

$=\mathrm{C}_{28} \mathrm{H}_{9} \mathrm{BrN}_{2} \mathrm{O}_{2}=\mathrm{C}_{16} \mathrm{H}_{4} \mathrm{BrNO}_{4}+\mathrm{C}_{12} \mathrm{H}_{7} \mathrm{~N}-\mathrm{H}_{2} \mathrm{O}_{2}$.

3) Phenyl-Chlorimesatim

$=\mathrm{C}_{28} \mathrm{H}_{9} \mathrm{ClN}_{2} \mathrm{O}_{2}=\mathrm{C}_{16} \mathrm{H}_{4} \mathrm{ClNO}_{4}+\mathrm{C}_{12} \mathrm{H}_{7} \mathrm{~N}-\mathrm{H}_{2} \mathrm{O}_{2}$.

1) Phenyl-Imesatì. $\mathrm{C}_{28} \mathrm{H}_{10} \mathrm{~N}_{2} \mathrm{O}_{2}$.

Diese Verbindung wird erhalten, wenn man 7,35 $\mathrm{Th}$. Isatin in einer geringen Menge Weingeist auflöst, alsdann zu dieser Lösung 4,65 Theile Anilin hinzufügt, bis zum Kochen erwärmt und zur Abkühlung hinstellt.

Nach einiger Zeit (zuweilen nach einigen Tagen, wenn nämlich $z u$ viel Weingeist angewandt und das Isatin, so wie das Anilin nicht in äquivalenter Menge genommen wurden) bildet sich in der erkalteten Flüssigkeit eine Menge gelber, nadelförmiger, zu Sternen gruppirter Krystalle. Die Mutterlauge, von diesen Krystallen abgegossen und eingedampft, giebt eine neue Menge, jedoch weniger reiner Krystalle. Aus 7,35 Grm. Isatin und 4,65 Grm. Anilin erhielt ich im Ganzen 10,5 Grm. PhenylImesatin.

Das auf diese Weise erhallene Phenyl-Imesatin wurde durch mehrmaliges Umkrystallisiren aus Weingeist gereinigt. Das Phenyl-Imesatin krystallisirt aus Weingeist in glänzenden, gelben, nadelförmigen, zu Sternen gruppirten Krystallen; unter der Lupe stellen diese Nadeln feine, durchsichtige, scharf zugespitzte Prismen dar. Sie lösen sich leicht in kochendem Weingeist, bedeutend schwerer in kaltem; die Lösung hat eine orangegelbe Farbe. In kochendem Wasser sind sie äusserst schwer löslich, die Lösung ist gelb gefärbt und setzt nach dem Erkalten Flocken $a b$, welche aus sehr feinen goldgelhen 
Nadeln bestehen. In Aether ist das Phenyl -.Imesatin löslich.

Beim Erhitzen auf Platinblech schmilzt es anfangs zu einer dunkelrothen Flüssigkeit, welche beim Erkalten zu einer amorphen Masse gesteht; dann zersetzt es sich; indem es viel Kohle hinterlässt und einen gelben Dampf entwickelt, der unangenehm auf die Athmungswerkzeuge wirkt.

Die weingeistige Lösung des Phenyl-Imesatin nimmt auf Zusatz von Salzsäure beim Kochen eine rothe Farbe an; beim Erkalten dieser Lösung setzt sich Isatin in Form rother, flacher Prismen $a b$ und in der Lösung bleibt salzsaures Anilin, welches leicht an der Reaction mit Schwefelsäure und saurem chromsauren Kali, so wie am Niederschlage mit Platinchlorid erkannt wird.

0,497 Grm. Phenyl-Imesatin gaben nach der Zersetzung mit Salzsäure 0,302 $\mathrm{Grm}$. Isatin.

In Salpetersäure löst es sich beim Erwärmen mit rother Farbe und zwar ohne Entwicklung rothbrauner Dämpfe. In starker Schwefelsäure löst es sich und bildet eine dunkelrothe Flüssigkeit, welche beim Verdünnen mit Wasser eine gelbe Farbe annimmt.

Beim Erwärmen mit starker wässriger Kalilösung nimmt es zuerst eine dunkelrothe Farbe an, nachher zersetzt es sich, indem es Anilin abscheidet und eine gelbe Lösung (von isatinsaurem Kali) bildet. Diese Lösung wird auf Zusatz von Salzsäure braun und giebt beim Abdampfen und nachherigen Abkühlen Isatin.

0,4013 Grm. Phenyl-Imesatin gaben beim Verbrennen $1,119 \mathrm{Grm}$. Kohlensäure und 0,184 Grm. Wasser.

0,3163 Grm. Phenyl-Imesatin gaben bei der Stickstoffbestimmung 0,290 Grm. Platin.

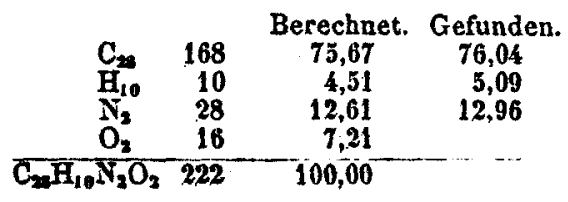


2) Phenyl-Bromimesatin. $\mathrm{C}_{28} \mathrm{H}_{9} \mathrm{BrN}_{2} \mathrm{O}_{2}$.

Um diese Verbindung zu bereiten, wurden 4,62 Grm.Bromisatin in kochendem Weingeist gelöst, zu dieser Lösung 1,86 Grm. Anilin gefügt, alles gekocht, etwas eingedampft und abkühlen gelassen. Es setzten sich nach dem Erkalten orangebraune Nadeln $a b$ und aus der Mutterlauge wurde beim Abdampfen eine neue Menge derselben erhalten; diese reinigte ich durch Umkrystallisiren aus Weingeist.

Das aus Weingeist krystallisirte Phenyl-Bromimesatin bildet schöne orangegelbe, seidenglänzende, flache Nadeln, welche sehr leicht in kochendem Weingeist löslich sind, dagegen sich in kaltem Weingeist weniger lösen; in kochendem Wasser sind sie beinahe ganz unlöslich, doch farbt sich hiebei das Wasser mit schwach gelber Farbe.

Die weingeistige Lösung des Phenyl-Bromimesatin, mit kochender Salzsäure behandelt, färbt sich roth und zersetzt sich in Bromisatin, welches sich beim Abkühlen der Lösung absetzt, und in Anilin, welches als salzsaures Salz in Lösung bleibt.

Beim Erwärmen mit wässriger Kalilösung färbt sich das Phenyl-Bromimesatin anfangs dunkelroth, nachher zersetzt es sich, scheidet Anilin ab, und es bildet sich eine gelbe Lösung (von bromisatinsaurem Kali), welche sich beim Erwärmen mit Salzsäure röthet und Bromisatin abscheidet.

0,392 Grm. der Substanz gaben beim Verbrennen 0,811 Grm. Kohlensäure und 0,125 Grm. Wasser.

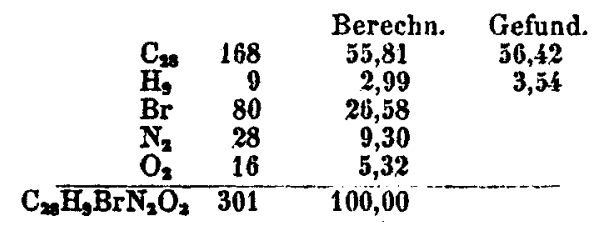

3) Phenyl-Chlorimesatin. $\mathrm{C}_{28} \mathrm{H}_{9} \mathrm{ClN}_{2} \mathrm{O}_{2}$.

Zur Darstellung dieser Verbindung wurden 3,5 Grm. Chlorisatin in 90-procentigem kochenden Weingeist gelöst, 
zu dieser Lösung 2,5 Grm. Anilin gefügt, alsdann erwärmt, etwas eingedampft und zur Abkühlung gestellt. Es setzten sich alsdann aus der Flüssigkeit rothbraune, scharf zugespitzte, flache Prismen ab, welche zu Bündeln gruppirt waren.

Das Phenyl-Chlorimesatin löst sich sehr leicht in kochendem Weingeist und auch ziemlich leicht in kaltem. Es krystallisirt aus stark und plötzlich abgekühltem Weingeist in orangegelben Nadeln, welche so sehr den Nadeln des Phenyl-Bromimesatin ähneln, dass man sie schwer zu unterscheiden vermag. Beim allmählichen Erkalten der weingeistigen Lösung des Phenyl-Chlorimesatin krystallisirt es in flachen zugeschärften rothbraunen Prismen. In Wasser ist es sehr schwer löslich, doch ertheilt es demselben eine gelbliche Farbc. Die weingeistige Lösung des PhenylChlorimesatin zersetzt sich durch Salzsäure in Chlorisatin und $\Lambda$ nilin. Mit einer wässrigen Kalilösung erwärmt, entbindet sich Anilin und es bildet sich eine gelbe Lösung (von chlorisatinsaurem Kali), welche sich beim Erwärmen mit Salzsäure röthet und Chlorisatin abscheidet.

0,343 Grm. der Substanz gaben beim Verbrennen 0,831 Grm. Kohlensăure und 0,122 Grm. Wasser.

\begin{tabular}{cccc} 
& & Bercehn. & Gefund. \\
$\mathrm{C}_{23}$ & 168 & 65,50 & 66,07 \\
$\mathrm{H}_{1}$ & 9 & 3,51 & 3,05 \\
$\mathrm{Cl}$ & 35,5 & 13,84 & \\
$\mathrm{~N}_{2}$ & 28 & 10,91 & \\
$\mathrm{O}_{2}$ & 16 & 0,21 & \\
\hline $\mathrm{C}_{22} \mathrm{H}_{2} \mathrm{C} 1 \mathrm{C}_{2} \mathrm{O}_{2}$ & 256,5 & 100,00 &
\end{tabular}

Ich versuchte ebenfalls, das Isatin mit Nitranilin und Tribromanilin zu copuliren, doch gelang mir dieses nicht; auch versuchte ich die Einwirkung des Chlorbenzoyl auf Imesatin, wobei beim Erwärmen sich Salzsäure entwickelte und der Rückstand, mit Kali behandelt, den Geruch des Benzonitrils und das Ansehen einer braunen harzartigen Masse batte.

Meines Erachtens ist die Reaction folgende:

$$
\frac{\mathrm{C}_{16} \mathrm{H}_{6} \mathrm{~N}_{2} \mathrm{O}_{2}}{\text { Imesatin. }}+\mathrm{C}_{44} \mathrm{H}_{3} \mathrm{O}_{2} \mathrm{Cl} \text { giebt } \frac{\mathrm{C}_{16} \mathrm{H}_{3} \mathrm{NO}_{4}}{\text { Isatin. }}+\mathrm{C}_{14} \mathrm{H}_{5} \mathrm{~N}+\mathrm{HCl} \text {. }
$$


Hierbei zersetzt sich das Isatin mit einem Ueberschuss des Chlorbenzoyls und bildet die harzige Masse.

Bei Erforschung der Einwirkung von Chlorbenzoyl auf Isatin bemerkte ich, dass bei schwacher Erwärmung das Isatin sich im Chlorbenzoyl auflöst und beim Erkalten ohne Veränderung herauskrystallisirt; beim heftigen Erwärmen aber entsteht eine Zersetzung, wobei sich eine schwarze kohlige Masse bildet; einmal sogar erhielt ich ein dunkelblaues krystallinisches Pulver, welches sich kaum in Weingeist löste.

\section{XXV}

Ueber die Einwirkung des Bromanilins und Chloranilins auf Isatin.

\section{Von}

A. Engelharat.

(Bullet. de St. Petersbourg.)

Die Analogie der chemischen Reactionen des Bromanilins und Chloranilins mit Anilin in Betracht ziehend, untersuchte ich die Einwirkung derselben auf Isatin und erhielt dabei Verbindungen, ähnlich dem Phenyl-Bromimesatin und Phenyl-Chlorimesatin, deren Beschreibung in der vorhergehenden Abhandlung: "Ueber die Einwirkung des Anilins auf Isatin, Bromisation und Chlorisatin "enthalten ist.

Diese Verbindungen, welche ich Chlorophenyl-Imesatin und Bromophenyl-Imesatin nenne, sind Copulationen des Chloranilins und Bromanilins mit Isatin, unter Ausscheidng sines Atoms Wasser $\left(\mathrm{H}_{2} \mathrm{O}_{2}\right)$ und $\mathrm{zwar}$ :

Bromophenyl-Imesatin

$$
=\mathrm{C}_{28} \mathrm{H}_{9} \mathrm{BrN}_{2} \mathrm{O}_{2}=\underbrace{\mathrm{C}_{16} \mathrm{H}_{5} \mathrm{NO}_{4}}_{\text {Isatin. }}+\underbrace{\mathrm{C}_{12} \mathrm{H}_{6} \mathrm{BrN}}_{\text {Bromanilin. }}-\mathrm{H}_{2} \mathrm{O}_{2} \text {. }
$$

